\title{
Emergence of pygmy dipole resonances: Magic numbers and neutron skins
}

\author{
Tsunenori Inakura, ${ }^{1}$ Takashi Nakatsukasa, ${ }^{1,2}$ and Kazuhiro Yabana ${ }^{2,1}$ \\ 1 RIKEN Nishina Center, Wako, 351-0198, Japan \\ ${ }^{2}$ Center for Computational Sciences, University of Tsukuba, Tsukuba 305-8571, Japan
}

\begin{abstract}
The pygmy dipole resonances (PDR) for even-even nuclei in $8 \leq Z \leq 40$ are studied performing a systematic calculation of the random-phase approximation with the Skyrme functional of SkM*. The calculation is fully self-consistent and does not assume any symmetry in the nuclear shape of the ground state. In every isotopic chain, the PDR emerges by showing a peak of the $E 1$ strength at energies less than $10 \mathrm{MeV}$. The $E 1$ strength of the PDR strongly depends on the position of the Fermi level and shows a clear correlation with the occupation of the orbits with the orbital angular momenta less than $3 \hbar(\ell \leq 2)$. We also found a strong correlation between the isotopic dependence of the neutron skin thickness and the pygmy dipole strength. The fraction of the energy weighted strength exhausted by the PDR and the neutron skin thickness show a linear correlation with the universal rate of about $0.2 \mathrm{fm}^{-1}$.
\end{abstract}

PACS numbers: 21.10.Pc, 21.60.Jz, 25.20.-x

Exotic nuclei show interesting new features in their response properties. The existence of loosely bound nucleons in the neutron skin and halo will significantly modify the strength distributions. Among them, the lowenergy electric dipole (E1) excitation, which emerges at the energy well below the giant dipole resonance (GDR), has been most extensively studied so far. These lowenergy peaks in the $E 1$ strength distribution are often called the pygmy dipole resonance (PDR). The PDR properties have a strong impact on astrophysical phenomena. The low-energy $E 1$ strengths in neutron-rich nuclei strongly enhance the radiative capture cross sections for low-energy neutrons, which directly affects the abundance distribution in the rapid neutron capture process [1]. In contrast to the GDR, the PDR is sensitive to nuclear properties at nuclear surface and at low density. Thus, its property may provide us with useful constraints on the energy density functional, to identify the equation of state (EOS) of the nuclear and neutron matters.

Another intriguing subject of interest is the relation between the PDR and the neutron skin thickness. The neutron skin thickness is known to be well correlated with the EOS of the neutron matter [2]. Thus, if the PDR is correlated with the neutron skin thickness, the investigation of the PDR may reveal the properties of the neutron stars, such as the proton ratio and radius. Piekarewicz suggested that the PDR strength is strongly correlated with the neutron skin thickness 3]. This means that the measurement of the PDR can provide information on the skin thickness. In contrast, a recent correlation analysis, which investigates the parameter dependence of various properties in ${ }^{208} \mathrm{~Pb}$, suggests a very weak correlation between the skin thickness and the PDR [4]. These conclusions were, however, obtained from the calculations for specific nuclei, such as spherical $\mathrm{Sn}$ isotopes and ${ }^{208} \mathrm{~Pb}$. It is thus desirable to perform a systematic and fully self-consistent calculation for the PDR for spherical and deformed nuclei, to reveal the nature of the PDR and its relation to the neutron skin.

The PDR were observed experimentally in neutron- rich nuclei such as $\mathrm{O}$ isotope [5, 6], ${ }^{26} \mathrm{Ne}$ 7], ${ }^{68} \mathrm{Ni} 8$ and $\mathrm{Sn}$ isotopes 9], but also in stable Ca [10], Sn 11, 12] and $\mathrm{Pb}$ isotopes [13, 14], and $N=82$ isotones [1519] as well. Although the nature of the PDR, including its relation to the neutron skin and halo, is still elusive, these experiments have identified the following properties of the PDR: The concentrated $E 1$ strengths have been observed around the threshold energy for the neutron separation. The energy-weighted strength for the PDR is less than $1 \%$ of the Thomas-Reiche-Kuhn (TRK) sumrule value in the stable nuclei and less than $5 \%$ in the case of neutron-rich nuclei.

Many microscopic studies based on the random phase approximation (RPA) with the Skyrme interaction 2025], the Gogny interaction 26, 27], and those based on the relativistic mean-field approach [3, 28 31] were carried out to investigate the PDR in the past decade. These microscopic calculations succeeded, to some extent, to reproduce dipole strength at low excitation. However, these microscopic calculations were applied to limited number of nuclei so far, and even basic questions, such as the condition on which the PDR appears in a nucleus, are not fully understood yet.

In the present paper, we report our systematic calculation of the $E 1$ response for a wide mass region with $8 \leq Z \leq 40$ (up to $A \sim 110$ ) in the self-consistent RPA with the Skyrme interaction, focusing on the emergence and the properties of the PDR. In the present calculation, we do not take into account the pairing correlation because we expect that its effect is not significant for the $E 1$ response in light- and medium-mass nuclei [3, 25]. We adopt the representation of the three-dimensional (3D) Cartesian grids. The real-space representation has an advantage over other basis representations, such as the harmonic oscillator basis, on the treatment of the continuum, since it is well controlled by the box size and can be directly compared with the continuum RPA calculations. We adopt the 3D adaptive coordinate grids [32] within a sphere of a radius $R_{\text {box }}=15 \mathrm{fm}$. The Skyrme functional of the $\mathrm{SkM}^{*}$ parameter set [33] is used. All the 
single-particle wave functions and potentials except for the Coulomb potential are assumed to vanish outside the sphere. The differentiation is approximated by a finite difference with the nine-point formula.

The first step of the investigation is to construct the Hartree-Fock ground state, using the imaginary-time method. Then, we solve the linear-response equation for the $E 1$ external field directly in the coordinate representation, avoiding explicit construction of unoccupied orbitals. Therefore, our calculation is free from a truncation for the unoccupied orbitals. Since the explicit construction of the RPA matrix in the 3D mesh representation is difficult, we use a new methodology, the finite amplitude method (FAM) 34], in evaluation of the residual field $\delta h$. The FAM allows us to construct $\delta h$ using the calculation of the Hamiltonian $h$ only. The residual field $\delta h$ in our calculations contains all terms of the Skyrme interaction, i.e., the residual spin-orbit interaction, the time-odd components, the residual Coulomb interaction, and so on. The linear-response equation is solved at given complex energies $\omega=E+i \gamma / 2$ using iterative solvers, such as the generalized conjugate residual (GCR) method. The calculations are performed at energies in spacing of $\Delta E=0.3 \mathrm{MeV}$ with a fixed imaginary part $0.5 \mathrm{MeV}$, corresponding to the smearing width $\gamma=1.0 \mathrm{MeV}$. Using the obtained RPA amplitudes, we compute the $E 1$ strength and the photoabsorption cross section. Details of the calculation can be found in Ref. [35].

The $E 1$ strength of even-even nuclei are calculated up to zirconium isotopes from the proton to neutron drip lines, except for nuclei with the neutron separation energy less than $2 \mathrm{MeV}$. This excludes the neutron dripline nuclei in which extended halos develop. In addition, the neutron-deficient nuclei from the proton drip-line to $N=50$ are also calculated for $40<Z \leq 50$. The total number of the calculated nuclides are 322: 40 spherical nuclei, 171 prolate nuclei, 56 oblate nuclei, and 55 triaxial nuclei.

The calculation shows that the PDR peaks appear in every isotopic chain, as demonstrated in Fig. 1 for $\mathrm{Ne}$ and $\mathrm{Ca}$ isotopes. Increasing neutron number with a fixed proton number, we see that the emergence of the PDR suddenly takes place at $N=16$ for $\mathrm{Ne}$ and at $N=$ 30 for $\mathrm{Ca}$ isotopes. In Ca isotopes, the PDR is rather distinctive and separated from the GDR peak. However, in the deformed neutron-rich Ne isotopes, it is not well separate from the low-energy tail of the GDR. Although it is not trivial how to define the PDR, the separate lowenergy peaks mostly appear at energies below $10 \mathrm{MeV}$. Thus, in this work, the pygmy dipole strength is defined by the $E 1$ strength at energies below $10 \mathrm{MeV}$.

We calculate fractions of the photoabsorption cross section $\sigma(E)$ integrated up to $E=10 \mathrm{MeV}$ to the integrated total cross section. This is equal to the ratio $m_{1}(\mathrm{PDR}) / m_{1}$ where $m_{1}(\mathrm{PDR})$ is the energy-weighted sum up to $10 \mathrm{MeV}$ and $m_{1}$ is the energy-weighted-sumrule value that is larger than the TRK value by $30 \sim 40$

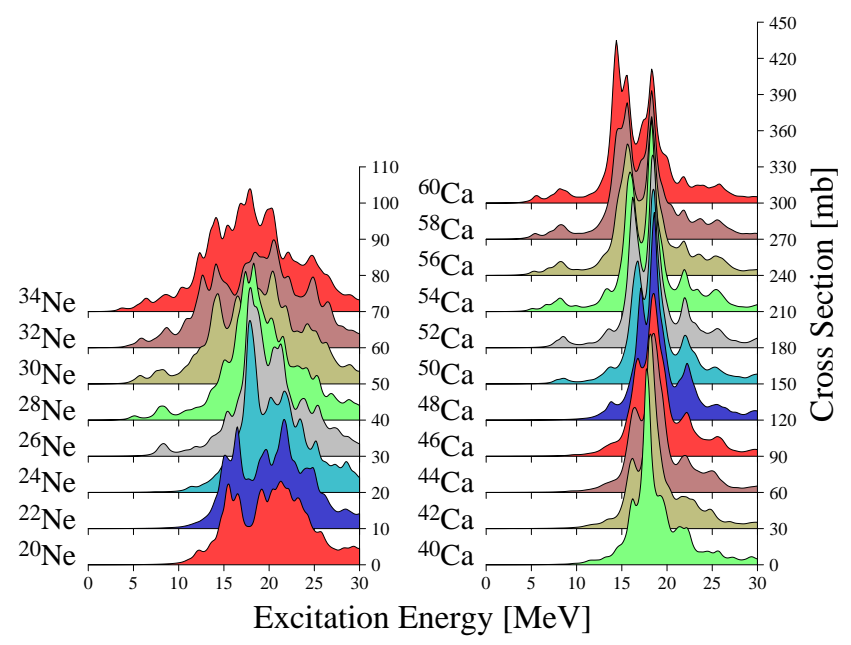

FIG. 1: (Color online) Calculated photoabsorption cross sections in $\mathrm{Ne}$ and $\mathrm{Ca}$ isotopes.

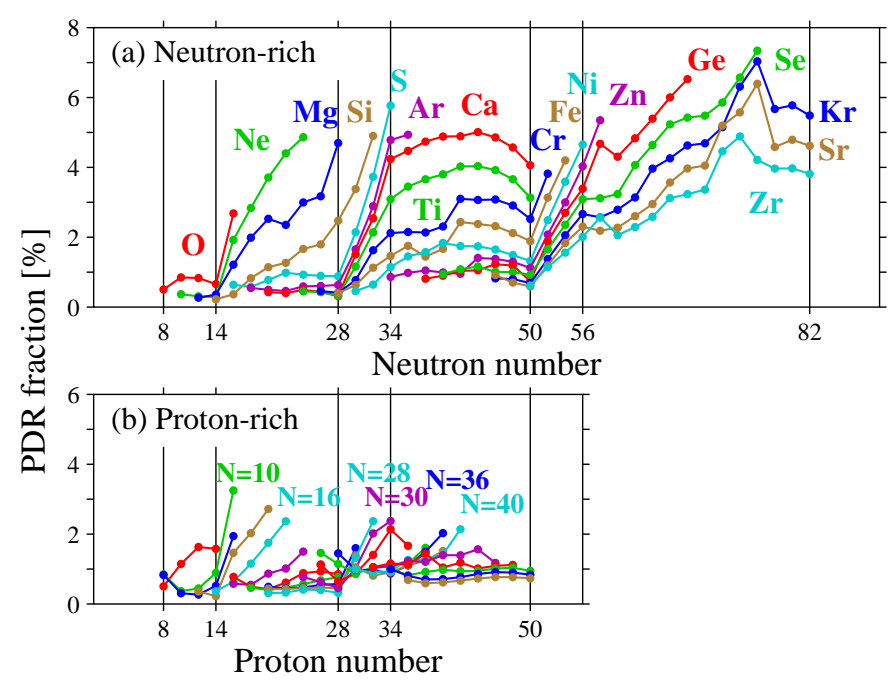

FIG. 2: (Color online) Fractions of photoabsorption cross sections $m_{1}(\mathrm{PDR}) / m_{1}$ for (a) stable and neutron-rich nuclei and for (b) stable and proton-rich nuclei, as functions of neutron number and proton number, respectively.

\%. The upper panel of Fig. 22 shows the PDR fractions of stable and neutron-rich nuclei as functions of neutron number. Here, we show isotopes with $8 \leq Z \leq 40$. In each isotopic chain, nuclei around stable region have small values of the fraction less than $\sim 1 \%$, consistent with the experimental data [5, 6, 10]. In these light nuclei, a prominent PDR does not appear in stable nuclei. Being away from the stable region, the fractions suddenly increase at specific neutron numbers: $N>14, N>28$, and $N>50$. These "magic" numbers for the emergence of the PDR indicate the presence of a strong shell effect of neutrons. The first clear indication of the kink structure appears at $N=14 \rightarrow 16$ for $\mathrm{O}, \mathrm{Ne}$ and $\mathrm{Mg}$ iso- 
topes. For these isotopes, the strength of the PDR has a strong correlation with the number of neutrons occupying the orbits with low orbital angular momenta (low- $\ell$ ), $s_{1 / 2}$ and $d_{3 / 2}(N=15 \sim 20)$. In case that the low- $\ell$ neutron orbits are weakly bound, they strongly expand in space due to the low centrifugal barrier. The kink is weakened by increasing the proton number and almost disappears for $\mathrm{Si}$ isotopes $(Z=14)$ in which $N=16$ corresponds to the stable nucleus ${ }^{30} \mathrm{Si}$. This should be due to the fact that these low- $\ell$ neutron orbits become more bound for nuclei with larger $Z$. The neutrons start filling the $f_{7 / 2}$ orbits at $N>20$, then, the growing rate of the PDR strength is reduced, which is most prominent for $\mathrm{Mg}$ isotopes.

The even more prominent kinks can be identified at the magic numbers of $N=28$ and $N=50$. The PDR fractions suddenly increase at $N=28 \rightarrow 30$ and continue to increase till $N=34$ where $2 p$ shell are filled. The increasing rate of the PDR fractions depends on the proton number. Namely, it is the largest for small- $Z$ isotopes, such as $\mathrm{Si}$ and $\mathrm{Ar}$ isotopes, while increasing $Z$ makes the rate smaller. Beyond $N=34$, the PDR fractions are roughly constant for $34<N \leq 50$, in which the neutrons are filling high- $\ell$ orbits of $f_{5 / 2}$ and $g_{9 / 2}$. They again show a sudden increase at $N=50 \rightarrow 52$, then, continue to linearly grow up until $2 d_{5 / 2}$ orbits are filled at $N=56$. Beyond that, it is difficult to see the definite trend, since the ordering of the orbits and the ground-state deformation change from nucleus to nucleus, depending on $Z$ and $N$. However, the careful investigation suggests that the occupation of $s_{1 / 2}$ and $d_{3 / 2}$ increases the PDR fraction, while that of $h_{11 / 2}$ reduces it. Thus, we may conclude that the spatially extended nature of the low- $\ell$ neutron orbits near the Fermi level plays a primary role for the the emergence and growth of the PDR. We have also observed that the deformation tends to increase the PDR strength, especially in the region $N>56$. This may be due to two effects; the mixture of the low- $\ell$ components in the orbits near the Fermi level and softening of the giant dipole resonance for the elongated direction.

In the proton-rich side, we can see the similar behaviors for the isotones with $8 \leq N \leq 50$ in the lower panel of Fig. 2, There are kinks in the PDR fractions at $Z=14$ and 28. However, the increase as a function of the proton number at $28<Z \leq 34$ is less prominent than that as a function of the neutron number at $28<N \leq 34$. This is because the Coulomb barrier prevents the low- $\ell$ proton orbits from spatial extension, especially in high- $Z$ nuclei.

Assuming the Steinwedel-Jensen model with the core and valence neutrons (protons), the PDR fraction is proportional to $N_{v} / N\left(Z_{v} / Z\right)$ where $N_{v}\left(Z_{v}\right)$ is the number of valence neutrons (protons) [36]. This is consistent with our result shown in Fig. 2 for valence nucleons defined as those in the low- $\ell$ orbits. However, the model predicts the PDR peak energy as $E_{\mathrm{PDR}} / E_{\mathrm{GDR}}=\sqrt{N_{v} / N}$, which disagree with our microscopic result. Figure 1 suggests that the PDR peak energy is rather insensitive to the number of nucleons.

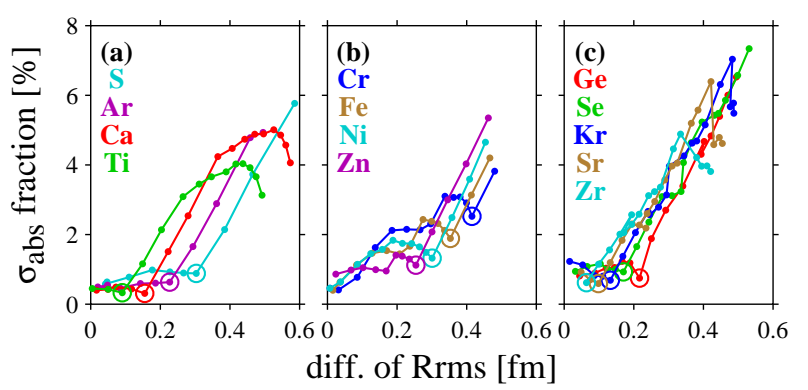

FIG. 3: (Color online) Correlations between fractions of photoabsorption cross section and neutron skin thickness. The kink positions at $N=28$ and 50 are denoted by circles. See text for details.

Next, let us examine the correlation between the PDR and the neutron skin thickness. The skin thickness is defined by the difference in radius between neutrons and protons: $\Delta R_{\mathrm{rms}} \equiv \sqrt{\left\langle r_{n}^{2}\right\rangle}-\sqrt{\left\langle r_{p}^{2}\right\rangle}$. We find that $\Delta R_{\mathrm{rms}}$ increases linearly with respect to the number of valence neutrons in the regions of $N=14-20, N=28-34$, and $N=50-56$, where the PDR fraction also shows significant linear increase as a function of the neutron number (Fig. 22). Plotting the PDR fraction as a function of $\Delta R_{\mathrm{rms}}$, we then observe a linear correlation between them. This is illustrated in Fig. 3 for isotopes with $Z=16 \sim 40$ which show the kinks at $N=28$ and 50 . The PDR fraction in each isotopic chain shows a linear correlation with the skin thickness in the regions of the neutron number $N=28-34$ and $N \geq 50$. The positions of the kinks are located at different values of $\Delta R_{\mathrm{rms}}$ for different isotopes. However, the slope is universal for all the isotopes; $0.18 \sim 0.20 \mathrm{fm}^{-1}$. It is remarkable to see that, even though the PDR fractions show no linear dependence on the neutron number at $N>56$ in Fig. 2 , they still keep the linear correlation with the skin thickness, until they start to decrease due to the dominant role of the $h_{11 / 2}$ orbits at $N \gtrsim 76$. Despite the fact that the deformation and shell ordering are different and vary from nucleus to nucleus, the universal linear correlation remains valid for $50 \leq N \lesssim 76$. It should be noted that the linear correlation can be observed only for each isotopic chain. Deleting the lines connecting isotopic chains in Fig. 3, we only see scattered points showing a weak correlation.

A detailed comparison with the available experimental data is beyond the scope of the present paper, since we use a smearing width of $\gamma=1 \mathrm{MeV}$ which prevents us from quantifying small low-energy $E 1$ strengths which carry less than $1 \%$ of the TRK sum value. So far, the observed PDR strength in nuclei with $Z \leq 40$ are very small (typically less than $1 \%$ of the TRK value), which is qualitatively consistent with our calculation because these nuclei are in the region before the kinks $(N \leq 14$, $28,50)$. The only exception is ${ }^{26} \mathrm{Ne}[7]$. The experimental data for ${ }^{26} \mathrm{Ne}$ indicates the PDR peak at $E_{\mathrm{PDR}} \approx 9 \mathrm{MeV}$ 
carrying about $5 \%$ of the TRK value. These values correspond to our calculated values of $E_{\mathrm{PDR}} \approx 8 \mathrm{MeV}$ with $2.6 \%$. The low-energy $E 1$ strength in ${ }^{68} \mathrm{Ni}$ is observed around $E=11 \mathrm{MeV}$ carrying about $5 \%$ of the TRK value [8]. Our calculation predict a PDR peak around $E=10 \mathrm{MeV}$ and $m_{1}(\mathrm{PDR}) / m_{1}$ indicates $4.6 \%$ of the TRK value where $m_{1}(\mathrm{PDR})$ is defined by the strength summed up to $E=12 \mathrm{MeV}$.

Finally, we comment on dependence of the results on the box size $R_{\text {box }}$ of the calculation. We have selected several nuclei to check how the result depends on $R_{\text {box }}$. The discretized continuum states, which depend on the choice of $R_{\text {box }}$, may affect the precise peak position and shape of the PDR, however, the PDR strength is very little affected. For instance, the enlargement of the $R_{\text {box }}$ to $25 \mathrm{fm}$ shifts the PDR fractions, $1.89 \% \rightarrow 1.94 \%$ for ${ }^{26} \mathrm{Ne}$ and $4.79 \% \rightarrow 4.65 \%$ for ${ }^{60} \mathrm{Ca}$. Thus, the features of the PDR discussed above are valid in the present calculation.

In summary, we carried out the systematic calculation of the low-lying $E 1$ strengths in even-even nuclei up to zirconium isotopes, including both spherical and deformed nuclei for the first time, by means of the fully self-consistent RPA with the Skyrme interaction utilizing the FAM. We found the PDR in every isotopic chain. The PDR strength in neutron-rich nuclei shows strong enhancement in $N>14$ for nuclei with $7<Z<13$, in $N>28$ for $13<Z<23$, and in $N>50$ for $23<Z \leq 40$. This suggests that the occupation of the low- $\ell$, such as $s, p$ and $d$ orbits, play a key role for the emergence of the PDR in neutron-rich nuclei. In the proton-rich side, the PDR strengths are hindered because of the Coulomb barrier to prevent those orbits from the spatial extension. The PDR distributions show a remarkable linear correlation with the neutron skin thickness in the regions of $28 \leq N \leq 34$ and $50 \leq N \lesssim 76$, with a universal slope of $0.18 \sim 0.20 / \mathrm{fm}$. The present result is consistent with the linear correlation reported in Ref. [3] for neutrondeficient $\mathrm{Sn}$ isotopes in the region of $56<N<70$, where the neutrons are filling the $s$ and $d$ orbits. Since heavy isotopes possess the neutron skin even in the neutrondeficient side, it is of great interest to perform a systematic study on the PDR in heavy systems.

This work is supported by the PACS-CS project of 09-a-25, 09-b-9, 10a-22, of the Center for Computational Sciences, University of Tsukuba and by the Large Scale Simulation Program No. 08-14, 09-16, and 09/10-10 of High Energy Accelerator Research Organization (KEK) and by Grant-in-Aid for Scientific Research on Innovative Areas (No. 20105003) and by the Grant-in-Aid for Scientific Research(B) (No. 21340073). The numerical calculations were performed on PACS-CS supercomputers in University of Tsukuba, on Hitachi SR11000 at KEK, and on the RIKEN Integrated Cluster of Clusters (RICC). We also thank the International Research Network for "Exotic Femto Systems" (EFES) of the Core-to-Core Programs of Japan Society for the Promotion of Science.
[1] S. Goriely, Phys. Lett. B 436, 10-18 (1998). S. Goriely et al., Nucl. Phys. A 706, 217-232 (2002); Nucl. Phys. A 739, 221-352 (2004).

[2] B. A. Brown, Phys. Rev. Lett. 85, 5296 (2000).

[3] J. Piekarewicz, Phys. Rev. C 73, 044325 (2006).

[4] P.-G. Reinhard and W. Nazarewicz, Phys. Rev. C 81, 051303(R) (2010).

[5] A. Leistenschneider et al., Phys. Rev. Lett. 86, 5442 (2001).

[6] E. Tryggestad et al., Phys. Lett. B 541, 52 (2002); Phys. Rev. C 67, 064309 (2003).

[7] J. Gibelin et al., Phys. Rev. Lett. 101, 212503 (2008).

[8] O. Wieland et al., Phys. Rev. Lett. 102, 092502 (2009).

[9] P. Adrich et al., Phys. Rev. Lett. 95, 132501 (2005).

[10] T. Hartmann et al., Phys. Rev. Lett. 85, 274 (2000); Phys. Rev. Lett. 93, 192501 (2004).

[11] K. Govaert et al., Phys. Rev. C 57, 2229 (1998).

[12] A. Klimkiewicz et al., Phys. Rev. C 76, 051603(R) (2007).

[13] N. Ryezayeva et al., Phys. Rev. Lett. 89, 272502 (2002).

[14] J. Enders et al., Nucl. Phys. A 724 (2003) 243.

[15] R.-D. Herzberg et al., Phys. Lett. B 390, 49 (1997); Phys. Rev. C 60, 051307 (1999).

[16] A. Zilges et al., Phys. Lett. B 542, 43 (2002); Nucl. Phys. A 731 (2004) 249-255.

[17] S. Volz et al., Nucl. Phys. A 779, 1 (2006).

[18] D. Savran et al., Phys. Rev. Lett. 97, 172502 (2006); Phys. Rev. Lett. 100, 232501 (2008).

[19] A.P. Tonchev et al., Phys. Rev. Lett. 104, 072501 (2010).
[20] G. Colò and P.F. Bortignon, Nucl. Phys. A 696, 427 (2001).

[21] D. Sarchi et al., Phys. Lett. B 601, 27 (2004).

[22] J. Terasaki and J. Engel, Phys. Rev. C 74, 044301 (2006).

[23] M. Matsuo et al., Phys. Rev. C 71, 064326 (2005).

[24] K. Yoshida and N. Van Giai, Phys. Rev. C 78, 064316 (2008). K. Yoshida, Phys. Rev. C 80, 044324 (2009).

[25] S. Ebata et al., Phys. Rev. C 82, 034306 (2010).

[26] S. Péru et al., Eur. Phys. J., A 26, 25 (2005).

[27] M. Martini et al., Phys. Rev. C 83, 034309 (2011).

[28] D. Vretenar et al.,Nucl. Phys. A 692, 496 (2001); Phys. Rev. C 63, 047301 (2001).

[29] N. Paar et al., Phys. Rev. C 67, 034312 (2003); Phys. Lett. B 606, 288 (2005); Phys. Rev. Lett. 94, 182501 (2005); Phys. Lett. B 624, 195 (2005); Phys. Rev. Lett. 103, 032502 (2009).

[30] Jun Liang et al., Phys. Rev. C 75, 054320 (2007).

[31] D. Peña Arteaga and P. Ring, Phys. Rev. C 77, 034317 (2008). D. Peña Arteaga et al., Phys. Rev. C 79, 034311 (2009).

[32] T. Nakatsukasa and K. Yabana, Phys. Rev. C 71, 024301 (2005).

[33] J. Bartel et al., Nucl. Phys. A 386 (1982) 79.

[34] T. Nakatsukasa et al., Phys. Rev. C 76, 024318 (2007).

[35] T. Inakura et al., Phys. Rev. C 80, 044301 (2009).

[36] Y. Suzuki et al., Prog. Theor. Phys. 83, 180 (1990). 